\title{
Re: Comparison of Single Photon Emission Computed Tomography Findings in Cases of Healthy Adults and Solvent-Exposed Adults
}

KEY WORDS: SPECT; toxic encephalopathy; solvents; pesticides; confounding; bias

The major thrust of the article by Fincher et al. [1997] appears to be an attempt to establish specific quantitative results of single photon emission computed tomography (SPECT) scans as criteria for differentiation of individuals with solvent-induced toxic encephalopathy from "normal" individuals. The article fails utterly in this attempt for a host of reasons, any one of which would be fatal to the effort. We highlight some of these reasons below.

1. The study purports to investigate 25 subjects with a history of mixed solvent exposure, and who met selection criteria which "paralleled the Swedish standard for clinical cases of solvent induced toxic encephalopathy." Five criteria are listed, and the article by Edling et al. [1990] is cited. In the article by Edling et al., all subjects had "at least 10 years of verified occupational exposure to organic solvents" ( $p$ 76). The mean durations of solvent exposure in the study by Edling et al. ranged from 23 to 26 years. However, the longest reported duration of alleged solvent exposure in the present study was for subject number 5, who was listed as having had only nine years of exposure. Fully 15 of the subjects were reported to have had exposure durations that were one year or less, seven of whom had alleged exposures lasting no more than two days!

Clearly, none of the "cases" chosen for the study met the criteria of Edling et al. [1990]. Such brief opportunities for exposure do not even come close to what is typically described in the medical literature among subjects with

Correspondence to: Dr. Franzblau, Dept. of Environmental and Industrial Health, University of Michigan School of Public Health, 109 Observatory Street, Ann Arbor, MI 48109-2029.

Accepted for publication 19 J une 1997 alleged chronic solvent-induced toxic encephalopathy. As in the study by Edling et al., solvent exposure histories described in such cases usually involve at least two or three decades in occupations which are known to provide opportunity for significant solvent exposure. Since none of the subjects in the present study met the exposure criteria for solvent-induced toxic encephalopathy, there is no scientific foundation for the conclusions in the study regarding SPECT findings for this clinical condition.

2. In addition to unacceptably short durations of exposure, many of the descriptions of "exposure incidents" (see Table IV) appear to have involved minimal, if any, exposure to solvents and thus are questionable in terms of producing any chronic neuropsychological effect (Case 16 - "Occupational exposure from office renovation" for one month; Case 17 - "Occupational exposure in a 'tight' or toxic building" for 1.5 years). Furthermore, we suspect that if properly questioned most of the "controls" in the study would also report similar histories of trivial opportunities for solvent exposures. Thus, using the authors' criteria for defining "exposures," the controls would not be truly unexposed. Hence, there is no foundation for alleging that differences in SPECT scan results are related to such exposures.

3. The 25 subjects selected as cases for inclusion in this study had their blood tested for a variety of solvents (see Table III for listing). All 25 cases reportedly had detectable levels of two or more of the chemicals for which testing was performed. These results are problematic for a number of reasons. First, the temporal relationship between the alleged exposures and time of blood sampling is not specified, making these results uninterpretable as biological indices of exposure to solvents, or measures of "bioaccumulation of solvents." Second, the concentrations of chemicals mea- 
sured in the blood are not stated. It is well known that all people in modern urban societies have detectable blood or breath levels of a wide variety of exogenous organic materials, including many of the chemicals listed in Table III of the article [see, for example, Ashley et al., 1996; Wallace et al., 1986, 1988]. Third, there is no indication that the controls were tested for the same chemicals. Moreover, the testing performed on the cases apparently was not blinded as to "case" vs. "control" status. There also appears to have been no consideration whatsoever to distinguishing markers of "exposure" from markers of "disease." Since the controls were not tested for the same organic chemicals in their blood, it is impossible to draw any conclusions as to whether differences in SPECT results are related to differences in blood solvent levels.

Furthermore, the descriptions under "Exposure incidents" are not consistent with the chemicals listed under "Solvents detected in blood" (see Table IV). For example, in Case 8 the "Exposure incident" involved trichloroethylene (TCE) used by a mechanic to clean parts, and yet, TCE is not listed under "Solvents detected in blood." Instead, six structurally different organic materials are listed (toluene; 1,1,1-trichloroethane; trimethylbenzenes; 2-methylpentane; 3-methylpentane; n-hexane). We see no relevance of these other solvents to the alleged exposure, and such results suggest that the solvent exposure incidents described in this report have no relationship to solvents measured in the blood. If the authors have a scientific rationale to tie the chemicals under "Exposure incidents" to "Solvents detected in blood," they should present this in the Methods or Discussion sections. Otherwise, data presented in Table IV are uninterpretable.

4. The authors intended to minimize the potential effects of extraneous medical and lifestyle variables (p 6, first column) on SPECT results. To do so they excluded from the study controls who had a history of concussions, head injuries, loss of consciousness, seizures, chronic pain conditions, illicit drug use, adverse reactions to household chemicals, or breast implants; and those who were current smokers, users of medications that altered cerebral blood flow, heavy consumers of caffeinated beverages, consumers of alcohol, and consumers of artificially sweetened beverages. This extraordinary list of exclusionary factors resulted in $74 \%$ of candidate controls being excluded from the study (in addition to an unstated number of candidates who were eliminated by a preliminary discussion with the investigators). What is most remarkable about this rigorous exclusionary policy is that none of these exclusionary criteria were applied to the cases. Thus, differences in SPECT results between the cases and the controls may be due to any combination of these factors that were admissible in cases and inadmissible in controls. This is an egregious example of selection bias, and would also exacerbate spectrum bias [see Ransohoff and Feinstein, 1978].

5. The authors apparently misunderstand the concept of confounding and sought to dismiss the possibility of confounding by age through statistical tests of significance [see Rothman, 1986; Hernberg, 1996]. On page 8 they discuss t-tests in which counts of late phase tracers among the youngest and oldest subjects were compared. It should be noted that the data presented by the authors in Table II show a $31 \%$ increase in SPECT counts with increasing age and that some of the t-tests were marginally significant until they were subjected to a Bonferroni correction procedure. The authors dismiss this obvious age effect, stating "These quantitative analyses suggest that correction for gender and age within the 25- to 45-year band is unnecessary." The issue they did not address, and which is central to the validity of their findings, is whether age confounds the association between SPECT results and case status. Here, they fail to provide the age distribution of the cases which would allow the reader to see if the cases and controls are of comparable ages, and they fail to perform any analyses in which age is controlled, which would reveal the extent to which the SPECT results are explained by age.

6. The article states that "Patients with detected levels of both pesticides and solvents were disqualified, due to the unknown neurotoxic effect of the pesticide" (p 9). The article does not provide any description of the panel of pesticides for which testing was performed. However, none of the cases were alleged to have had clinically significant exposure to pesticides, so the scientific relevance and application of this exclusion criterion is unclear. Also, many pesticides are well known to be ubiquitous in the environment, and to be measurable in the blood of almost all humans on the planet (e.g., dichlorodiphenyltrichloroethane, or DDT, or its metabolic derivatives). Was detection of DDT, or DDT metabolites, used as an exclusionary criterion? Which pesticides, or other chemicals, were used to exclude subjects and, most significantly, why didn't the controls have similar measurements performed (it is probable that controls would have demonstrated a similar pattern of results)?

7. SPECT testing was not performed or reported in an interpretable manner. In the current paper, SPECT data analysis was performed using raw counts of reconstructed images. Raw counts of Tc-99m HMPAO SPECT images, however, do not necessarily represent quantitatively either cerebral blood flow or brain metabolism, due to the following reasons. After intravenous injection, the tracer is diluted in the venous system and cardiac chambers. Even though a similar amount of Tc-99m HMPAO (mean $26.1 \mathrm{mCi}$ ) was injected, arterial input concentration to the brain would have been variable across subjects because of normal variation in the initial distribution volumes which are independent from 
either cerebral blood flow or metabolism. There was no measurement of arterial concentration of the injected tracer nor proper metabolite measurement provided in the current study, which makes quantitative estimation of cerebral blood flow or metabolism impossible.

Some authors normalize raw counts to reference regions (such as a global count) to partially overcome the lack of such measurements [e.g., Payne et al., 1996], but this approach was not used in the current study. In addition, early images obtained during the first two minutes contain not only cerebral blood flow information but also cerebral vascular blood volume and some back-diffusion from radioactivity delivered earlier within the first two minutes. A delay between the start of injection to the arrival of the injected tracer to the brain would also be variable among subjects, which exerts an additional uncertainty in pixel raw counts on early images.

Raw counts on delayed images are similarly affected with the initial plasma concentration of the tracer, which is independent from either cerebral blood flow or metabolism and radioactivity in the vascular blood volume as well. SPECT image acquisition and reconstruction methods were not described sufficiently (i.e., the number of projections, attenuation correction method), which makes replication of the current study difficult. Quantitative analysis of cerebral blood flow measured with Tc-99m HMPAO has to take account of such multiple factors carefully [e.g., Murase et al., 1992], and has been discussed vigorously in various settings in the research community [e.g., Rattner et al., 1991]. Unfortunately, the analysis employed in the current paper does not involve any "quantitative" technique in this regard nor provide information necessary to estimate specific parameters such as cerebral blood flow. In addition, "region-of-interest" analysis used in this study was not described specifically (e.g., what part of the "frontal" lobe is included in analyses?). When estimating hemispheric or global counts, simple averages of multiple regions were calculated (Tables I, II, and VII). Consequently, the averaged values were biased by smaller structures, such as the basal ganglia and thalamus, whose values are more prone to partial volume effects on SPECT images. Hence, conclusions in the present paper based on functional brain SPECT imaging results are suspect and cannot be used as a basis for concluding that there are abnormalities in any patient group.

In summary, this study was poorly designed and executed and the results are presented inadequately. There are numerous flaws that create confounding or bias and the conclusions are not supported by the data presented. In our opinion, the weaknesses in this study are so serious and glaringly obvious that it cannot be relied upon for any medical or scientific purpose.
Alfred Franzblau, MD
Dept. of Environmental and Industrial Health, University of Michigan School of Public Health
Satoshi Minoshima, MD, PhD
Dept. of Internal Medicine,
University of Michigan School of Medicine
Thomas G. Robins, MD, MPH
Dept. of Environmental and Industrial Health, University of Michigan School of Public Health
David H. Garabrant, MD, MPH
Dept. of Environmental and Industrial Health, University of Michigan School of Public Health Ann Arbor, MI

\section{REFERENCES}

Ashley DL, Bonin MA, Cardinali FL, McCraw JM, Wooten JV (1996): Measurement of volatile organic compounds in human blood. Environ Health Perspec 104:871-877.

Edling C, Ekberg K, Ahlborg G Jr, Alexandersson R, Barregard L, Ekenvall L, Nilsson L, Svensson BG (1990): Long-term follow up of workers exposed to solvents. Br J Ind Med 47:75-82.

Fincher CE, Chang T, Harrell EH, Kettelhut MC, Rea WJ, Johnson A, Hickey DC, Simon TR (1997): Comparison of single photon emission computed tomography findings in cases of healthy adults and solventexposed adults. Am J Ind Med 31:4-14.

Hernberg S (1996): Significance testing of potential confounders and other properties of study groups - misuse of statistics. Scand J Work Environ Health 22:315-316.

Murase K, Tanada S, Fujita H, Sakaki S, Hamamoto K (1992): Kinetic behavior of technetium-99m-HMPAO in the human brain and quantification of cerebral blood flow using dynamic SPECT. J Nucl Med 33:135-143.

Payne JK, Trivedi MH, Devous MD Sr (1996): Comparison of technetium99m-HMPAO and xenon-133 measurements of regional cerebral blood flow by SPECT. J Nucl Med 37:1735-1740.

Ransohoff DF, Feinstein AR (1978): Problems of spectrum and bias in evaluating the efficacy of diagnostic tests. NEJM 299:926-930.

Rattner Z, Smith EO, Woods S, Dey J, Hoffer PB (1991): Toward absolute quantitation of cerebral blood flow using technetium-99m-HMPAO and a single scan. J Nucl Med 32:1506-1507.

Rothman, KJ (1986): "Modern Epidemiology." Boston: Little, Brown \& Company, pp 180-181.

Wallace L, Pellizzari E, Hartwell T, Zelon H, Sparacino C, Perritt R, Whitmore R (1986): Concentrations of 20 volatile organic compounds in the air and drinking water of 350 residents of New Jersey compared with concentrations in their exhaled breath. J Occup Med 28:603-608.

Wallace LA, Pellizzari ED, Hartwell TD, Whitmore R, Zelon H, Perritt R, Sheldon L (1988): The California team study: Breath concentrations and personal exposures to 26 volatile compounds in air and drinking water of 188 residents of Los Angeles, Antioch, and Pittsburgh, CA. Atmospheric Environ 22:2141-2163. 